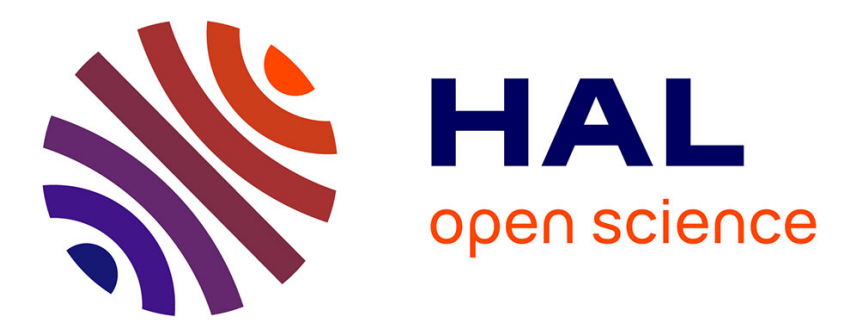

\title{
Iterative Poisson-Gaussian Noise Parametric Estimation for Blind Image Denoising
}

Anna Jezierska, Jean-Christophe Pesquet, Hugues Talbot, Caroline Chaux

\section{To cite this version:}

Anna Jezierska, Jean-Christophe Pesquet, Hugues Talbot, Caroline Chaux. Iterative Poisson-Gaussian Noise Parametric Estimation for Blind Image Denoising. IEEE International Conference on Image Processing, Oct 2014, Paris, France. pp.1-5. hal-01060081v2

\section{HAL Id: hal-01060081 https://hal.science/hal-01060081v2}

Submitted on 18 Sep 2014

HAL is a multi-disciplinary open access archive for the deposit and dissemination of scientific research documents, whether they are published or not. The documents may come from teaching and research institutions in France or abroad, or from public or private research centers.
L'archive ouverte pluridisciplinaire HAL, est destinée au dépôt et à la diffusion de documents scientifiques de niveau recherche, publiés ou non, émanant des établissements d'enseignement et de recherche français ou étrangers, des laboratoires publics ou privés. 


\section{ITERATIVE POISSON-GAUSSIAN NOISE PARAMETRIC ESTIMATION FOR BLIND IMAGE DENOISING}

\author{
A. Jezierska, J.-C. Pesquet, H. Talbot* \\ Univ. Paris-Est \\ LIGM UMR-CNRS 8049 \\ 5 bd Descartes, \\ 77454 Marne-la-Vallée, France \\ \{first.last\}@univ-paris-est.fr
}

C. Chaux

\author{
Aix-Marseille Univ. \\ LATP UMR CNRS 7353 \\ 39 rue F. Joliot-Curie, \\ 13453 Marseille, France \\ caroline.chauxelatp.univ-mrs.fr
}

\begin{abstract}
This paper deals with noise parameter estimation from a single image under Poisson-Gaussian noise statistics. The problem is formulated within a mixed discrete-continuous optimization framework. The proposed approach jointly estimates the signal of interest and the noise parameters. This is achieved by introducing an adjustable regularization term inside an optimized criterion, together with a data fidelity error measure. The optimal solution is sought iteratively by alternating the minimization of a label field and of a noise parameter vector. Noise parameters are updated at each iteration using an Expectation-Maximization approach. The proposed algorithm is inspired from a spatial regularization approach for vector quantization. We illustrate the usefulness of our approach on macroconfocal images. The identified noise parameters are applied to a denoising algorithm, so yielding a fully automatic denoising scheme.
\end{abstract}

Index Terms - Poisson-Gaussian noise, parameter estimation, Expectation-Maximization, proximal algorithms, discrete optimization.

\section{INTRODUCTION}

Estimation of noise parameters is a classical problem in signal and image processing. For instance, Donoho [1] proposed to employ a robust Gaussian variance estimator from the wavelet coefficients of an image, known as the median absolute deviation estimator (MAD). Since then, many alternative methods addressing this problem have been proposed [2], [3], [4], possibly from a Bayesian perspective, e.g. in [5]. A more general problem was investigated in [6] where the authors consider the mixture of a white Gaussian noise and a random impulsive noise.

Unfortunately, in many cases, the imaging noise is not simply additive. Due to the quantum nature of light, the images are degraded by shot noise. Thermal noise may also play a prominent role in some modalities, e.g. astronomical imaging. Although a Gaussian approximation is sufficient in some cases, one may need to consider a noise model where the mean or the variance depends on the image intensity. Consequently, studies related to image recovery in the presence of signal dependent noise constitutes an active research area. Examples include restoration methods in the presence of Poisson noise [7] and more recently Poisson-Gaussian models [8], [9].

\footnotetext{
* This work was supported by the Agence Nationale de la Recherche under grant ANR-09-EMER-004-03.
}

However, the associated noise identification problems have not yet been extensively studied in the literature.

In this paper, we address the problem of estimating the parameters of a Poisson-Gaussian noise from a single image. The problem is formulated within a variational framework. The proposed alternating optimization algorithm jointly estimates the signal of interest and the noise parameters from a single noise realization. We consider various choices of regularization functions promoting sparsity, including nonconvex ones.

The estimation of noise parameters in the presence of multiplicative or Poisson noise is more challenging than for additive stationary Gaussian noise. The additional challenge stems from the noise nonstationarity. There have been many attempts to address this issue, for instance in the context of CCD imaging [10], radar [11], and confocal microscopy [12]. However, all these approaches share a similar structure. Namely, they proceed by first determining pixel groups with uniform intensities [13], [14] followed by a parameter fitting procedure. Similarly to most of these techniques, our approach includes both a segmentation and an estimation process. However, unlike the previous works, the two main steps are repeated iteratively. At each iteration, the new partition and noise parameter estimates are obtained by minimizing a cost function.

The remaining of this paper is organized as follows. The model is introduced in Section 2. Then, the considered parameter estimation problem and the proposed methodological and numerical solutions are described in Section 3. We provide experimental results in Section 4, where the performance of our approach is illustrated using macroconfocal data. Finally, Section 5 concludes the paper.

\section{MODEL}

Let $x$ be the original image of size $N \times M$. Its values are assumed to belong to a finite set $\left\{u_{1}, \ldots, u_{K}\right\}$ where $K \in \mathbb{N}^{*}$ is the number of distinct intensity values. We will denote by $u$ the vector $\left[u_{1}, \ldots, u_{K}\right]^{\top} \in \mathbb{R}^{K}$. We consider a partition $\mathbb{P}=\left(\mathbb{D}_{k}\right)_{1 \leq k \leq K}$ of the image support $\mathbb{S}=\{1, \ldots, N\} \times\{1, \ldots, M\}$, which is related to the image $x$ through the following relation:

$$
x=\left(u_{i_{\mathbb{P}}(\mathbf{s})}\right)_{\mathbf{s} \in \mathbb{S}} \in\left\{u_{1}, \ldots, u_{K}\right\}^{N \times M},
$$

where $\left(i_{\mathbb{P}}(\mathbf{s})\right)_{\mathbf{s} \in \mathbb{S}} \in \mathbb{I}=\{1, \ldots, K\}^{N \times M}$ is a label image defined as

$$
(\forall \mathbf{s} \in \mathbb{S})(\forall k \in\{1, \ldots, K\}) \quad i_{\mathbb{P}}(\mathbf{s})=k \Leftrightarrow \mathbf{s} \in \mathbb{D}_{k}
$$


Since $x$ is a function of $i_{\mathbb{P}}$ and $u$, it will be denoted by $x_{i_{\mathbb{P}}, u}$ throughout this paper.

The observed noisy image $y \in \mathbb{R}^{N \times M}$ is a realization of a random vector $Y$ and is such that

$$
y=\alpha q+w
$$

where $\alpha$ is a positive scaling factor, and $q=(q(\mathbf{s}))_{\mathbf{s} \in \mathbb{S}}($ resp. $w)$ are realizations of mutually independent random vectors $Q$ (resp. $\left.W=(W(\mathbf{s}))_{\mathbf{s} \in \mathbb{S}}\right)$ with independent components. More precisely,

$$
\begin{aligned}
Q & \sim \mathcal{P}\left(x_{i_{\mathbb{P}}, u}\right) \\
(\forall \mathbf{s} \in \mathbb{S}) \quad W(\mathbf{s}) & \sim \mathcal{N}\left(c, \sigma^{2}\right),
\end{aligned}
$$

where $c \in \mathbb{R}$ and $\sigma \in(0,+\infty)$ are the mean and the standard deviation of the Gaussian noise component, respectively, and $\mathcal{P}(x)$ denotes a multivariate Poisson distribution with mean vector $x$.

Then, the parameter estimation problem corresponds to finding a vector of unknown noise parameters $\theta \in \mathcal{T}$, where $\theta=$ $\left[c, \sigma^{2}, \alpha, u^{\top}\right]^{\top}$ and $\mathcal{T}$ is the parameter set which is here equal to $\mathbb{R} \times(0,+\infty)^{2} \times C, C$ being a closed convex subset of $\mathbb{R}^{K}$. The unknown vector $\theta$ is related to the vector of observations $y$ through the probability density function of $Y$, which has the following form: for every $y=(y(\mathbf{s}))_{\mathbf{s} \in \mathbb{S}} \in \mathbb{R}^{N \times M}$,

$p_{Y}\left(y ; i_{\mathbb{P}}, \theta\right)=\prod_{k=1}^{K} \prod_{\mathbf{s} \in \mathbb{D}_{k}}\left(\sum_{q(\mathbf{s})=0}^{+\infty} \frac{e^{-u_{k}}\left(u_{k}\right)^{q(\mathbf{s})}}{q(\mathbf{s}) !} \frac{e^{-\frac{(y(\mathbf{s})-\alpha q(\mathbf{s})-c)^{2}}{2 \sigma^{2}}}}{\sqrt{2 \pi \sigma^{2}}}\right)$.

\section{PROPOSED APPROACH}

\subsection{Problem formulation}

We adopt a variational approach where the parameter vector $\theta$ is estimated by minimizing a penalized criterion. We then solve

$$
\underset{\left(i_{\mathbb{P}}, \theta\right) \in \mathbb{I} \times \mathcal{T}}{\operatorname{minimize}} \Phi\left(\theta, i_{\mathbb{P}}, y\right)+\rho\left(i_{\mathbb{P}}\right) .
$$

The above function $\Phi$ is defined as the neg-log-likelihood of $y$ :

$$
\Phi\left(\theta, i_{\mathbb{P}}, y\right)=-\log \left(p_{Y}\left(y ; i_{\mathbb{P}}, \theta\right)\right)
$$

and $\rho$ is a regularization function aiming at incorporating a priori information about the homogeneity of the level sets of the image. Note that Problem (7) is nonconvex due to the fact that $i_{\mathbb{P}}$ belongs to a (nonconvex) set of discrete values.

\subsection{Algorithm}

We propose the following alternating optimization algorithm:

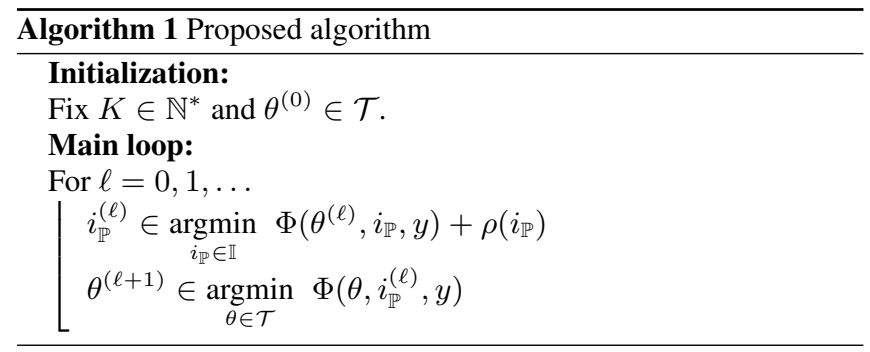

Assuming that noise parameters $\left(c, \sigma^{2}, \alpha\right)$ are known, the above algorithm reduces to the joint denoising-quantization algorithm proposed in [15]. In particular, the first step of Algorithm 1, which consists of searching $i_{\mathbb{P}}$, is unchanged. Classical discrete optimization techniques are used for this purpose [16]. Additional difficulties arise in the second step of Algorithm 1 since the minimized function is no longer convex. However, we are able to use some of the results of [17], where the problem of estimating Poisson-Gaussian noise parameters from time series is addressed. The proposed method however required a large enough number of image realizations in order to provide consistent estimates of the parameters.

\subsection{Parameter update}

In order to solve numerically the nonconvex problem stated in the second step of Algorithm 1, we propose to employ the ExpectationMaximization (EM) algorithm [18]. In such settings, the EM algorithm generates a sequence of estimates $\left(\theta^{(\ell)}\right)_{\ell \in \mathbb{N}}$ which is given by

$$
(\forall \ell \in \mathbb{N}) \quad \theta^{(\ell+1)}=\underset{\theta \in \mathcal{T}}{\operatorname{argmin}} \Phi\left(\theta \mid \theta^{(\ell)}\right)
$$

where

$$
\begin{aligned}
\Phi\left(\theta \mid \theta^{(\ell)}\right)= & \frac{1}{2 \sigma^{2}} \sum_{\mathbf{s} \in \mathbb{S}} \mathrm{E}_{Q \mid Y=y, \theta^{(\ell)}}\left[(y(\mathbf{s})-\alpha Q(\mathbf{s})-c)^{2}\right] \\
& +\frac{N M}{2} \ln \left(\sigma^{2}\right)+\sum_{k=1}^{K} \operatorname{card}\left(\mathbb{D}_{k}^{(\ell)}\right) u_{k} \\
& -\sum_{k=1}^{K} \ln u_{k} \sum_{\mathbf{s} \in \mathbb{D}_{k}^{(\ell)}} \mathrm{E}_{Q \mid R=r, \theta^{(\ell)}}[Q(\mathbf{s})]
\end{aligned}
$$

and $(Q(\mathbf{s}))_{\mathbf{s} \in \mathbf{S}}$ are the components of $Q$ as defined in (4) with $i_{\mathbb{P}}=$ $i_{\mathbb{P}}^{(\ell)}$.

This leads to the following operations to be performed at each iteration $\ell$ :

1. Find $u^{(\ell+1)}$ as a solution of

$$
u^{(\ell+1)} \in \underset{u \in \mathbb{R}^{K}}{\operatorname{argmin}} \vartheta(u)
$$

where $\vartheta(u)=\sum_{k=1}^{K} \vartheta_{k}\left(u_{k}\right)$, and, for every $k \in\{1, \ldots, K\}$,

$$
\vartheta_{k}\left(u_{k}\right)=\operatorname{card}\left(\mathbb{D}_{k}^{(\ell)}\right) u_{k}-\ln u_{k} \sum_{\mathbf{s} \in \mathbb{D}_{k}^{(\ell)}} \mathrm{E}_{Q \mid R=r, \theta^{(\ell)}}[Q(\mathbf{s})] .
$$

In the unconstrained case when $C=\mathbb{R}^{K}$, we have then

$$
u^{(\ell+1)}=\frac{\sum_{\mathbf{s} \in \mathbb{D}_{k}^{(\ell)}} \mathrm{E}_{Q \mid R=r, \theta^{(\ell)}}[Q(\mathbf{s})]}{\operatorname{card}\left(\mathbb{D}_{k}^{(\ell)}\right)} .
$$

On the other hand, for some regularization terms [15], set $C$ is useful to impose a total order constraint. This amounts to minimizing $\vartheta$ under the constraints that $V u \in D=$ $\left[\delta,+\infty\left[\times\left[0,+\infty\left[^{K-1}\right.\right.\right.\right.$ where $\delta$ is some small positive value, and $V$ is the linear operator defined as

$$
\begin{aligned}
& V: \mathbb{R}^{K} \rightarrow \mathbb{R}^{K} \\
& \quad\left(u_{1}, \ldots, u_{K}\right) \mapsto\left(u_{1}, u_{2}-u_{1}, \ldots, u_{K}-u_{K-1}\right) .
\end{aligned}
$$


2. Determine $c^{(\ell+1)}$ and $\alpha^{(\ell+1)}$ by solving the following system of linear equations:

$$
\begin{gathered}
{\left[\begin{array}{cc}
N M & \sum_{\mathbf{s}} \mathrm{E}_{Q \mid Y=y, \theta^{(\ell)}}[Q(\mathbf{s})] \\
\sum_{Q \mid Y=y, \theta^{(\ell)}}[Q(\mathbf{s})] & \sum_{\mathbf{s}} \mathrm{E}_{Q \mid Y=y, \theta^{(\ell)}}\left[Q(\mathbf{s})^{2}\right]
\end{array}\right]\left[\begin{array}{l}
c^{(\ell+1)} \\
\alpha^{(\ell+1)}
\end{array}\right]} \\
=\left[\begin{array}{c}
\sum_{\mathbf{s}} y(\mathbf{s}) \\
\sum_{\mathbf{s}} y(\mathbf{s}) \mathrm{E}_{Q \mid Y=y, \theta^{(\ell)}}[Q(\mathbf{s})]
\end{array}\right] \cdot
\end{gathered}
$$

3. Compute $\left(\sigma^{2}\right)^{(\ell+1)}$ as

$$
\frac{\sum_{\mathbf{s}} y(\mathbf{s})\left(y(\mathbf{s})-\alpha^{(\ell+1)} \mathrm{E}_{Q \mid Y=y, \theta^{(\ell)}}[Q(\mathbf{s})]-c^{(\ell+1)}\right)}{N M} .
$$

Note that even when a closed form solution to Problem (11) does not exist, it can still be solved efficiently using proximal tools. We propose to solve numerically this problem by using a primal-dual proximal algorithm [19].

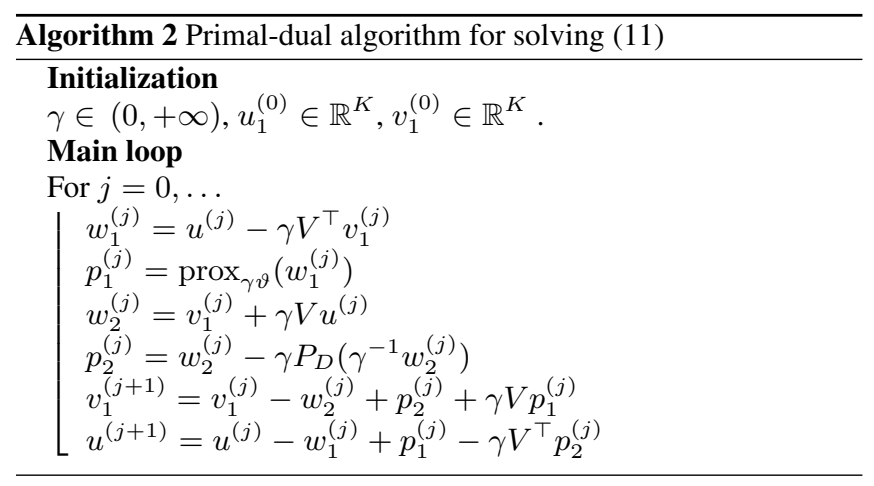

In the above algorithm, we denote the projector onto the closed convex set $D$ by $P_{D}$. The proximity operator of $\gamma \vartheta$ with $\gamma>0$ admits a closed form expression [20] and it is given by

$$
\begin{aligned}
\left(\forall u=\left(u_{k}\right)_{1 \leq k \leq K}\right. & \left.\in \mathbb{R}^{K}\right) \\
& \operatorname{prox}_{\gamma \vartheta}(u)=\left(\operatorname{prox}_{\gamma \vartheta_{k}}\left(u_{k}\right)\right)_{1 \leq k \leq K}
\end{aligned}
$$

where, for every $k \in\{1, \ldots, K\}$,

$$
\begin{aligned}
& \operatorname{prox}_{\gamma \vartheta_{k}}\left(u_{k}\right)=\frac{1}{2}\left(u_{k}-\operatorname{card}\left(\mathbb{D}_{k}\right)\right. \\
&\left.+\sqrt{\left|u_{k}-\operatorname{card}\left(\mathbb{D}_{k}\right)\right|^{2}+4 \sum_{\mathbf{s} \in \mathbb{D}_{k}} \mathrm{E}_{Q \mid R=r, \theta}(\ell)}[Q(\mathbf{s})]\right)
\end{aligned}
$$

The convergence of the primal-dual algorithm is guaranteed, using the following result deduced from [19, Theorem 4.2].

Theorem 3.1 Under the assumptions that $\gamma \in[\epsilon,(1-\epsilon) / \beta]$ where $\epsilon \in(0,1 /(\beta+1))$ and $\beta=\|V\|$, there exists a minimizer $\widehat{u}$ of $(11)$ such that the sequences $\left(u^{(j)}\right)_{j \in \mathbb{N}}$ and $\left(p_{1}^{(j)}\right)_{j \in \mathbb{N}}$ converge to $\widehat{u}$.
Concerning the computation of the required conditional expectation values, it can be shown that, for every $\mathbf{s} \in \mathbb{S}$,

$$
\mathrm{E}_{Q \mid Y=y, \theta^{(\ell)}}[Q(\mathbf{s})]=\frac{\zeta_{\mathbf{s}}^{(\ell)}}{\eta_{\mathbf{s}}^{(\ell)}} \quad \text { and } \quad \mathrm{E}_{Q \mid Y=y, \theta^{(\ell)}}\left[Q(\mathbf{s})^{2}\right]=\frac{\xi_{\mathbf{s}}^{(\ell)}}{\eta_{\mathbf{s}}^{(\ell)}}
$$

where

$$
\begin{aligned}
& \zeta_{\mathbf{s}}\left(\theta^{(\ell)}\right)=\sum_{q(\mathbf{s})=0}^{\infty} \Pi_{\mathbf{s}}\left(\theta^{(\ell)}, 1, q(\mathbf{s})\right) \\
& \eta_{\mathbf{s}}\left(\theta^{(\ell)}\right)=\sum_{q(\mathbf{s})=0}^{\infty} \Pi_{\mathbf{s}}\left(\theta^{(\ell)}, 0, q(\mathbf{s})\right) \\
& \xi_{\mathbf{s}}\left(\theta^{(\ell)}\right)=\sum_{q(\mathbf{s})=0}^{\infty} \Pi_{\mathbf{s}}\left(\theta^{(\ell)}, 1, q(\mathbf{s})\right)+\sum_{q(\mathbf{s})=0}^{\infty} \Pi_{\mathbf{s}}\left(\theta^{(\ell)}, 2, q(\mathbf{s})\right)
\end{aligned}
$$

In these formulas, for every $(d, q(\mathbf{s})) \in \mathbb{N}^{2}$,

$$
\begin{array}{r}
\Pi_{\mathbf{s}}(\theta, d, q(\mathbf{s}))=\exp \left(-\frac{(y(\mathbf{s})-\alpha(q(\mathbf{s})+d)-c)^{2}}{2 \sigma^{2}}\right) \\
\times \frac{\left(u_{i_{\mathbb{P}}^{(\ell)}(\mathbf{s})}\right)^{q(\mathbf{s})+d}}{q(\mathbf{s}) !} .
\end{array}
$$

The infinite summations in (20), (21) and (22) are approximated by finite sums with the following bounds:

$$
q(\mathbf{s})^{+}=q(\mathbf{s})^{*}+\Delta \frac{\sigma}{\alpha}, \quad q(\mathbf{s})^{-}=q(\mathbf{s})^{*}-\Delta \frac{\sigma}{\alpha}
$$

where $q(\mathbf{s})^{*}=\frac{\sigma^{2}}{\alpha^{2}} \mathrm{~W}\left(\frac{\alpha^{2}}{\sigma^{2}} u_{i_{\mathbb{P}}^{(\ell)}(\mathbf{s})} e^{\frac{\alpha}{\sigma^{2}}(y(\mathbf{s})-c-d \alpha)}\right)$. Here, $\mathbf{W}$ denotes the Lambert function and $\Delta>0$. This choice has been shown to ensure a fast decay of the approximation error as a function of $\Delta$.

\subsection{Patch-based initialization}

Since Problem (7) is nonconvex, the proposed approach is sensitive to initialization. In the following, we propose an initialization procedure based on image patches [21]. Firstly, the observed image $y$ is decomposed into non overlapping patches $\Theta y=\left\{\Theta y_{1}, \ldots, \Theta y_{O}\right\}$, where $O$ stands for the total number of patches. Next, we estimate the vectors mean $(\Theta y) \in \mathbb{R}^{O}$ and $\operatorname{Var}(\Theta y) \in \mathbb{R}^{O}$, whose elements are the mean and the variance over the pixels belonging to each patch, respectively. Under the assumption that the intensity of each patch is constant, the mean and variance of the patch is given by: for every $o \in\{1, \ldots, O\}$,

$$
\begin{aligned}
& {[\operatorname{mean}(\Theta y)]_{o}=\alpha v_{o}+c} \\
& {[\operatorname{var}(\Theta y)]_{o}=\alpha^{2} v_{o}+\sigma^{2}}
\end{aligned}
$$

where $v_{o} \in\left\{u_{1}, \ldots, u_{K}\right\}$. Although, the assumption about the constant intensity of each patch is not exactly satisfied, an approximation $\theta^{(0)}$ of the parameter vector is given by

- $\alpha^{(0)}=[\operatorname{Var}(\Theta y)]_{o_{1}} /[\text { mean }(\Theta y)]_{o_{1}}$, where $o_{1}=\operatorname{argmax}_{o \in\{1, \ldots, O\}}[\text { mean }(\Theta y)]_{o}$

- $c^{(0)}=\min _{o \in\{1, \ldots, O\}}[\operatorname{mean}(\Theta y)]_{o}$

- $\left(\sigma^{2}\right)^{(0)}=[\operatorname{Var}(\Theta y)]_{o_{2}}$, where $o_{2}=\operatorname{argmin}_{o \in\{1, \ldots, O\}}[\text { mean }(\Theta y)]_{o}$. 
- $u_{1}^{(0)}=\epsilon$, where $\epsilon$ is a small value greater than 0 , and $(\forall k \in$ $\{2, \ldots, K\}) u_{k}^{(0)}=u_{k-1}^{(0)}+u_{\max } /(K-1)$. The maximum intensity value $u_{\max }$ of $x$ is assumed to be known.

Note that in contrast with the approach proposed in [17] the EM algorithm is initialized with an improved set of parameters at each iteration.

\section{RESULTS}

We evaluate the robustness of our model under different working conditions. For all the presented experiments, the patch size is $12 \times$ $12, K=20$, and $\epsilon=0.2$. The regularization $\rho$ is an anisotropic total variation-like function defined as follows: let $(n, m)$ denote the coordinates of a generic pixel $\mathbf{s}$,

$$
\begin{aligned}
& \rho\left(i_{\mathbb{P}}\right)=\mu\left(\sum_{n=1}^{N-1} \sum_{m=1}^{M} \psi\left(\left|i_{\mathbb{P}}(n+1, m)-i_{\mathbb{P}}(n, m)\right|\right)\right. \\
& \left.+\sum_{n=1}^{N} \sum_{m=1}^{M-1} \psi\left(\left|i_{\mathbb{P}}(n, m+1)-i_{\mathbb{P}}(n, m)\right|\right)\right), \quad \mu \geq 0 .
\end{aligned}
$$

We set the regularization parameter $\mu$ to 1.3 when $\psi$ is set to a binary cost function defined as

$$
(\forall \xi \in[0,+\infty)) \quad \psi(\xi)= \begin{cases}0 & \text { if } \xi=0 \\ 1 & \text { otherwise }\end{cases}
$$

and 0.8 when $\psi$ is the identity function.

The results of the time series noise identification procedure described in [17] provide a high quality image, akin to a ground truth for real microscopy data (Figs $1(\mathrm{a}, \mathrm{e})$ ). In order to show the versatility of our model, we use $\psi$ defined as the identity function for the experiments presented in Figs 1 (a-d), while the binary cost function (27) is used for the experiment illustrated in Figs 1 (e-h). For the identity function, a total order constraint needs to be enforced in the EM step. For these two experiments, $u_{\max }$ is set to 150 and 40 respectively. In the first case, the image in Fig. 1 (a) was corrupted by Poisson-Gaussian noise with parameters $\alpha=5, c=150$, and $\sigma^{2}=125$. The identified noise parameters were $\widehat{\alpha}=4.89$, $\widehat{c}=156.35$, and $\widehat{\sigma}^{2}=115.2$ (Fig. 1 (c)). In the second case of Fig. 1 (e) the estimated value of $\alpha=20, c=150$, and $\sigma^{2}=1000$ were $\widehat{\alpha}=19.5, \widehat{c}=142$, and $\widehat{\sigma}^{2}=907$ (Fig. 1 (g)).

In Fig. 1, the estimated noise parameters are subject to small remaining errors with respect to the true values. The quantized image, a side result of our procedure, is also displayed. These results show that our procedure provides reliable results on real data that can be fed to a denoising procedure, e.g. [8]. The corresponding PSNR values are $28.45 \mathrm{~dB}$ and $28.82 \mathrm{~dB}$ for the images in Figs $1(\mathrm{~d})$ and $1(\mathrm{~h})$, respectively. For comparison, and to emphasize the importance of accurate parameter estimation, we averaged the PSNR of 50 image denoising realizations. These had parameters set around the optimal value, i.e we allowed parameters to vary following a Gaussian distribution with a standard deviation of $10 \%$ of the optimal value. This resulted in PSNRs of only $26.43 \mathrm{~dB}$ and $26.20 \mathrm{~dB}$, respectively.

\section{CONCLUSIONS}

We have proposed a reliable Poisson-Gaussian noise parameter estimation method for imaging applications, which only requires a single noisy image as input. The derived alternating minimization procedure allows us to estimate the noise parameters required by many denoising procedures. Here we used the method proposed in [8]. However, other denoising procedures can be used as well. The simulation results demonstrate the usefulness of our approach on confocal images. To our knowledge, this is the first blind estimation method for handling Poisson-Gaussian noise with nonzero background. In our future work, we plan to extend the proposed image noise estimation approach and to investigate its use for other parametric forms of complex noise probability distributions.
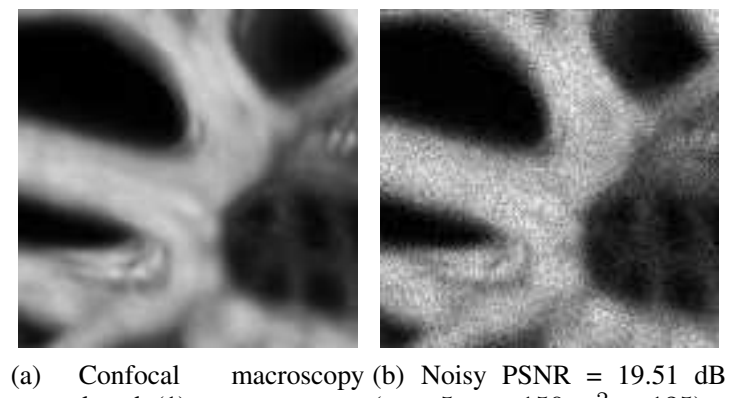
ground truth (1) $\left(\alpha=5, c=150, \sigma^{2}=125\right)$
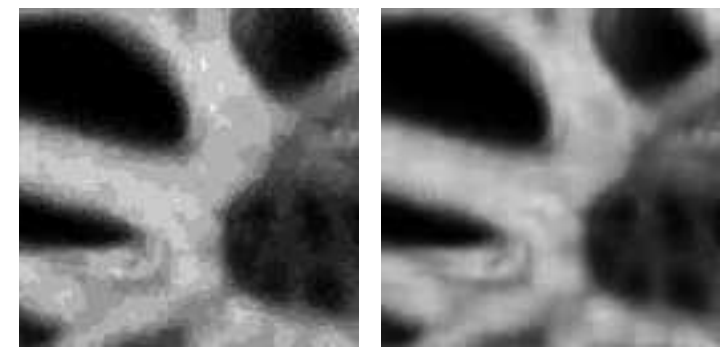

(c) Noise parameter iden. ( $\widehat{\alpha}=(\mathrm{d})$ Denoising result $4.89, \widehat{c}=156.35, \widehat{\sigma}^{2}=115.2$ ) PSNR $=28.45 \mathrm{~dB}$
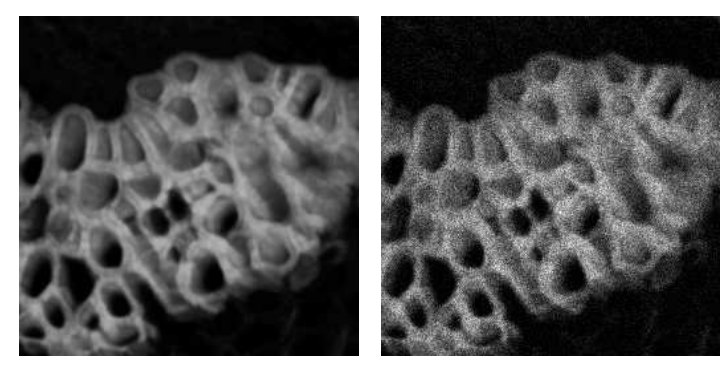

(e) Confocal ground truth (2)

macroscopy (f) Noisy PSNR=19.35 ( $\alpha=$

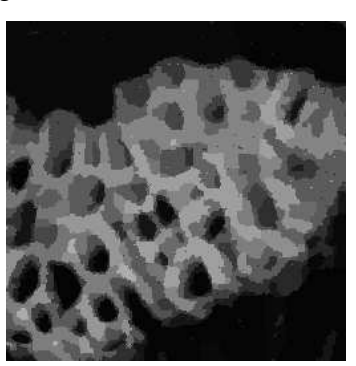
$\left.20, c=150, \sigma^{2}=1000\right)$

(g) Noise parameter iden. ( $\widehat{\alpha}=(\mathrm{h})$ Denoising result $\left.19.5, \widehat{c}=142, \widehat{\sigma}^{2}=907\right) \quad$ PSNR $=28.82 \mathrm{~dB}$

Fig. 1. Single image noise identification results.

Acknowledgement We thank Florian Luisier for providing us with the PURE-LET code. 


\section{REFERENCES}

[1] D. Donoho, "Denoising by soft-thresholding," IEEE Trans. Inform. Theory, vol. 41, pp. 613-627, 1995.

[2] A. De Stefano, P. R. White, and W. B. Collis, "Training methods for image noise level estimation on wavelet components.," EURASIP J. Adv. Sig. Proc., vol. 2004, no. 16, pp. 2400-2407, Jan. 2004.

[3] W. Liu, T. Liu, M. Rong, R. Wang, and H. Zhang, "A fast noise variance estimation algorithm," in Asia Pacific Conference on Postgraduate Research in Microelectronics and Electronics (PrimeAsia), Macau, China, Oct. 2011, pp. 61 -64.

[4] S. Abramov, V. Abramova, V. Lukin, and K. Egiazarian, "Robust local scale estimators for blind evaluation of noise variance in images," in 5th International Symposium on Communications Control and Signal Processing (ISCCSP), Rome, Italy, May 2012, pp. $1-4$.

[5] N. Dobigeon, A. O. Hero, and J.-Y. Tourneret, "Hierarchical Bayesian sparse image reconstruction with application to MRFM," IEEE Trans. on Image Processing, vol. 18, no. 9, pp. 2059-2070, Sept. 2009.

[6] R. Grou-Szabo and T. Shibata, "A dominant-noise discrimination system for images corrupted by content-independent noises without a priori references," in 5th International Conference on Signal Processing and Communication Systems (ICSPCS), Honolulu, Hawaii, Dec. 2011, pp. 1 -6.

[7] N. Pustelnik, C. Chaux, and J.-C. Pesquet, "Parallel proximal algorithm for image restoration using hybrid regularization," IEEE Transactions on Image Processing, vol. 20, no. 9, pp. 2450-2462, Sept. 2011.

[8] F. Luisier, T. Blu, and M. Unser, "Image denoising in mixed PoissonGaussian noise," IEEE Transactions on Image Processing, vol. 20, no. 3, pp. 696-708, Mar. 2011.

[9] A. Jezierska, E. Chouzenoux, J.-C. Pesquet, and H. Talbot, "A primal-dual proximal splitting approach for restoring data corrupted with Poisson-Gaussian noise," Kyoto, Japan, Mar. 2012, pp. 1085-1088.

[10] A. Foi, M. Trimeche, V. Katkovnik, and K. Egiazarian, "Practical Poissonian-Gaussian noise modeling and fitting for singleimage raw-data," IEEE Transactions on Image Processing, vol. 17, pp. 1737-1754, Oct. 2008.

[11] V.V. Lukin, N.N. Ponomarenko, S.K. Abramov, B. Vozel, K. Chehdi, and J. T. Astola, "Filtering of radar images based on blind evaluation of noise characteristics," in Proc. of SPIE, Image and Signal Processing for Remote Sensing XIV, L. Bruzzone, C. Notarnicola, and F. Posa, Eds. 2008, vol. 7109, pp. $1-12$, SPIE.

[12] P. Paul, H. Duessmann, T. Bernas, H. Huber, and D. Kalamatianos, "Automatic noise quantification for confocal fluorescence microscopy images," Computerized Medical Imaging and Graphics, vol. 34, no. 6, pp. 426-434, Sep. 2010.

[13] S. Abramov, V. Zabrodina, V. Lukin, B. Vozel, K. Chehdi, and J. Astola, "Improved method for blind estimation of the variance of mixed noise using weighted LMS line fitting algorithm," in Proceedings of 2010 IEEE International Symposium on Circuits and Systems (ISCAS), Paris, France, Jun. 2010, pp. 2642-2645.
[14] Z Liao, S. Hu, M. Li, and W. Chen, "Noise estimation for single-slice sinogram of low-dose X-ray computed tomography using homogenous patch," Mathematical Problems in Engineering, vol. 2012, pp. 16 pages, Jul. 2012.

[15] C. Chaux, A. Jezierska, J.-C. Pesquet, and H. Talbot, “A spatial regularization approach for vector quantization," Journal of Mathematical Imaging and Vision, vol. 41, pp. 23-38, Dec. 2011.

[16] K. Murota, "Algorithms in discrete convex analysis," IEICE Transactions on Systems and Information, vol. E83-D, pp. 344-352, Mar. 2000.

[17] A. Jezierska, C. Chaux, J.-C. Pesquet, H. Talbot, and G. Engler, "An EM approach for time-variant Poisson-Gaussian model parameter estimation," IEEE Transactions on Signal Processing, , vol. 62, no. 1, pp. 17-30, 2014.

[18] A. P. Dempster, N. M. Laird, and D. B. Rubin, "Maximum likelihood from incomplete data via the EM algorithm," Journal of the Royal Statistical Society, B, vol. 39, pp. 1-38, 1977.

[19] P. L. Combettes and J.-C. Pesquet, "Primal-dual splitting algorithm for solving inclusions with mixtures of composite, Lipschitzian, and parallel-sum type monotone operators," SetValued and Variational Analysis, vol. 20, pp. 307-330, Aug. 2012.

[20] P. L. Combettes and J.-C. Pesquet, "Proximal splitting methods in signal processing," in Fixed-point algorithms for inverse problems in science and engineering, $\mathrm{H}$. $\mathrm{H}$. Bauschke, $\mathrm{R}$. Burachik, P. L. Combettes, V. Elser, D. R. Luke, and H. Wolkowicz, Eds., pp. 185-212. Springer Verlag, 2010.

[21] J. Salmon, C-A. Deledalle, R. Willett, and Z. T. Harmany, "Poisson noise reduction with Non-Local PCA," in International Conference on Acoustics, Speech, and Signal Processing (ICASSP), Kyoto, Japan, Mar. 2012, pp. 1109-1112. 